\title{
Avaliação do efeito antinociceptivo e da toxicidade aguda do extrato aquoso da Hyptis fruticosa Salmz. ex Benth.
}

\author{
Aline B.L. Silva, Kellyane S. Dias, Maxsuel S. Marques, Igor A.C. Menezes, Thiago C. Santos, \\ Iderjane C.M. Mello, Ana Carla C.D. Lisboa, Sócrates C.H. Cavalcanti, Rosilene M. Marçal*, \\ Angelo R. Antoniolli
}

\author{
Departamento de Fisiologia, CCBS, Universidade Federal de Sergipe, Av. Marechal Rondon, S/N, Rosa
} Elze, 49100-000, São Cristóvão, Sergipe, Brasil

\begin{abstract}
RESUMO: Este trabalho descreve o efeito antinociceptivo e a toxicidade aguda do extrato aquoso das folhas da Hyptis fruticosa Salmz. ex Benth. (Lamiaceae). O extrato aquoso liofilizado, administrado por via oral, reduziu as contorções abdominais induzidas por ácido acético $(200,400$ e $500 \mathrm{mg} / \mathrm{kg})$ e o tempo de reação dos animais na primeira fase do teste da formalina $(100 \mathrm{mg} / \mathrm{kg}$ e $400 \mathrm{mg} / \mathrm{kg}$ ). No teste da placa quente, o extrato aquoso aumentou o tempo de latência ao calor (100 e $200 \mathrm{mg} / \mathrm{kg}$ ) tendo este efeito sido revertido pelo antagonista opióide naloxona $(5 \mathrm{mg} / \mathrm{kg}$; i.p.). No ensaio de toxicidade aguda, não foi detectada a morte de nenhum animal após tratamento com doses de até $5 \mathrm{~g} / \mathrm{kg}$ (v.o.) do extrato. Em conclusão, os resultados obtidos indicam que o extrato aquoso da Hyptis fruticosa apresenta efeito antinociceptivo em camundongos e não apresenta toxicidade aguda nas doses testadas.
\end{abstract}

Unitermos: Hyptis fruticosa, Lamiaceae, antinociceptivo, toxicidade aguda, extrato aquoso.

\begin{abstract}
Evaluation of the analgesic effect and acute toxicity of the aqueous extract of Hyptis fruticosa (Salmz. ex Benth.)". The antinociceptive effect and the acute toxicity of Hyptis fruticosa leaves were evaluated through the administration of its aqueous extract in mice. The extract, administered orally $(200,400$, and $500 \mathrm{mg} / \mathrm{kg})$, reduced the nociceptive response in the writhing test as well as in the early phase of the formalin test $(100$ and $400 \mathrm{mg} / \mathrm{kg}$ ) and it increased the latency time in the hot plate test $(100$ and $200 \mathrm{mg} / \mathrm{kg}$ ). The antinociceptive effect was reversed by naloxone $(5 \mathrm{mg} / \mathrm{kg}$, i.p.). Moreover, no animal deaths were observed in doses up to $5 \mathrm{~g} / \mathrm{kg}$. In conclusion, the aqueous extract of Hyptis fruticosa showed no acute toxicity at the evaluated doses and revealed antinociceptive effect in mice. Such effects are possibly associated with the opioid system activation.
\end{abstract}

Keywords: Hyptis fruticosa, Lamiaceae, antinociceptive, aqueous extract, acute toxicity.

\section{INTRODUÇÃO}

O gênero Hyptis, constituído por mais de 300 espécies, exibe a maior diversidade morfológica na região do Cerrado Brasileiro (Harley, 1988). Suas espécies são aromáticas e freqüentemente usadas no tratamento de infecções gastrintestinais, cãibras e dor, assim como infecções de pele (Septimio, 1994; Morais et al., 2005). Atividades antifúngica (De Oliveira et al., 2004), antibacteriana (Souza et al., 2003), antiulcerogênica (Barbosa; Ramos, 1992), larvicida (Costa et al., 2005), e antidepressiva (Bueno et al., 2006) dentre outras, têm sido reportadas para plantas do gênero Hyptis. Os efeitos antinociceptivo e antiinflamatório foram avaliados apenas para o extrato aquoso das folhas de Hyptis pectinata (Bispo et al., 2001; Falcão et al., 2005).

Hyptis fruticosa Salzm ex Benth (Lamiaceae) é um arbusto que se encontra amplamente distribuído na região Nordeste do Brasil (De Almeida; Albuquerque, 2002). No estado de Sergipe, esta planta é popularmente conhecida por "Alecrim de Tabuleiro" sendo a infusão de suas folhas popularmente utilizada no combate à dor e na desinfecção de pisos. No entanto, a Hyptis fruticosa tem sido objeto de poucos estudos científicos podendo-se citar apenas atividade antibacteriana observada na presença do extrato metanólico da raiz de H. fruticosa (De Araujo et al., 1974), o isolamento de três diterpenóides deste extrato (Marletti et al., 1976; Delle Monache et al., 1977) e atividades antibacteriana e antineoplásica detectada na presença de quinonóides isolados do extrato metanólico da raiz da H. fruticosa (De Araujo et al., 1974).

Apesar do uso popular da Hyptis fruticosa no combate à dor, não foram encontrados na literatura estudos que investigassem a eficácia analgésica desta planta. Considerando este fato, o objetivo deste trabalho foi avaliar o efeito analgésico sugerido pelo uso popular. A toxicidade aguda foi também investigada.

MATERIAL E MÉTODO 


\section{Material botânico}

Foram utilizadas folhas frescas de Hyptis fruticosa coletadas na cidade de São Cristóvão (1056'S; $\left.37^{\circ} 05^{\prime} \mathrm{W}\right)$, Sergipe, Brasil. A identidade botânica da planta foi confirmada pelo biólogo Prof. Dr. Adauto Souza Ribeiro e um exemplar coletado encontra-se depositado no Herbário da Universidade Federal de Sergipe (número ASE01137; Universidade Federal de Sergipe, CCBS, Departamento de Biologia, São Cristovão, Sergipe, Brasil).

\section{Animais}

Foram utilizados camundongos Swiss (20-35 g) machos e fêmeas e os grupos de animais foram separados com pesos aproximados. Os animais foram mantidos em caixas plásticas com comida e água ad libitum, tendo sido submetidos à privação de ração durante as seis horas que precederam os experimentos. Os protocolos utilizados foram aprovados pelo Comitê de Ética em Pesquisa com Animais (CEPA) desta instituição (processo no. 38/05) e os experimentos foram realizados de acordo com as normas vigentes para trabalho com animais de laboratório e com as normas éticas para investigações de dor experimental em animais acordados (Zimmermann, 1983).

\section{Preparo do extrato}

As folhas da planta foram secas em estufa (40 ${ }^{\circ} \mathrm{C}$; $48 \mathrm{~h}$ ) e pulverizadas em moinho de facas. A infusão do pó das folhas foi preparada utilizando-se 70 gramas do pó por litro de água destilada $\left(100^{\circ} \mathrm{C}\right)$. Após filtração sob pressão reduzida, a solução obtida foi liofilizada (Liofilizador Benchtop, Vir Tis, USA) para a obtenção do extrato seco $(7,4 \% ; 10,4 \mathrm{~g})$.

\section{Estudo de toxicidade aguda $\left(\mathrm{DL}_{50}\right)$}

A toxicidade aguda em camundongos $(n=5)$ foi avaliada através da administração oral de doses crescentes do extrato aquoso $(1,3$ e $5 \mathrm{~g} / \mathrm{kg})$ ou de igual volume do veículo (água) e verificação do número de óbitos após 48 horas (Lorke, 1983).

Estudos de analgesia. Teste de contorções abdominais induzidas pelo ácido acético (Koster et al., 1959)

Os animais $(\mathrm{n}=9)$ foram tratados com o extrato aquoso da planta nas doses de 200,400 e $500 \mathrm{mg} / \mathrm{kg}$ (v.o.) ou com igual volume de água (grupo controle; v.o.). Sessenta minutos após cada tratamento, foi administrado o ácido acético $(0,6 \% ; 0,1 \mathrm{~mL}$ de solução/ $10 \mathrm{~g}$ de animal; i.p.). As contorções abdominais foram contadas durante vinte minutos, iniciando-se dez minutos após a administração de ácido acético. O fármaco padrão indometacina (10 $\mathrm{mg} / \mathrm{kg}$; i.p.; 45 minutos antes da administração do ácido acético) foi utilizada como controle positivo.

\section{Teste da formalina (Dubuisson; Dennis, 1977)}

Os animais foram tratados com o extrato aquoso da planta (100, 200 e $400 \mathrm{mg} / \mathrm{kg}$; v.o.), ou com igual volume de água destilada (v.o.). Sessenta minutos após cada tratamento, os camundongos $(\mathrm{n}=7)$ receberam uma injeção intraplantar de formalina a $1 \%(20 \mu \mathrm{L} /$ animal $)$. Após a administração da formalina, o tempo (s) que o animal passou lambendo a pata traseira esquerda foi cronometrado em duas fases (0-5 min primeira fase; 20-30 min; segunda fase), sendo considerado tempo zero o momento imediatamente após a administração da formalina. $\mathrm{O}$ fármaco padrão morfina $(7,5 \mathrm{mg} / \mathrm{kg}$, i.p., 45 min antes da formalina) foi utilizada como controle positivo.

\section{Teste da placa quente (Ankier, 1974)}

Os animais $(\mathrm{n}=7)$ foram colocados sobre uma placa de alumínio aquecida $\left(55 \pm 0,5{ }^{\circ} \mathrm{C}\right)$ e o tempo (s) que cada animal gastou para retirar uma pata traseira da placa de alumínio e levá-la à boca (tempo de latência) foi cronometrado. Foram realizadas medidas repetidas nos tempos 0 e +15 sendo o tempo zero instituído como sendo 60 minutos após o tratamento com extrato aquoso $(100,200$ e $400 \mathrm{mg} / \mathrm{kg}$; v.o.) ou com igual volume de água destilada (v.o.) e o tempo +15 foi instituído como 15 minutos após o tempo zero. Com o objetivo de se evitar danos às patas dos animais foi estabelecido um tempo de corte de 30 segundos. Morfina (7,5 mg/kg; i.p.) foi administrada 45 antes do tempo zero e naloxona $(5 \mathrm{mg} /$ $\mathrm{kg}$; i.p.) foi administrada 20 minutos antes da morfina. Um grupo de animais foi co-tratado com o extrato aquoso da Hyptis fruticosa (200 mg/kg; v.o.) e com naloxona (5 $\mathrm{mg} / \mathrm{kg}$; i.p.).

\section{Análise matemática dos dados}

Os resultados foram expressos através de média \pm erro padrão da média ( $\mathrm{M} \pm$ E.P.M.). As diferenças entre os grupos foram determinadas através da análise de variância ANOVA de uma via, seguida pelo teste de Bonferroni quando apropriado. Um nível de significância de $5 \%$ foi utilizado.

\section{RESULTADOS}

No teste das contorções abdominais (Tabela 1), o extrato aquoso da Hyptis fruticosa, nas doses de 200, 400 e $500 \mathrm{mg} / \mathrm{kg}$ (v.o.), reduziu de forma significativa as contorções abdominais induzidas por ácido acético $(0,6 \%)$. O fármaco padrão indometacina (10 mg/kg; i.p.), no entanto, apresentou maior eficácia do que o extrato aquoso neste teste.

O agonista opióide morfina (7,5 mg/kg; i.p.), de 
Tabela 1. Efeito do extrato aquoso da Hyptis fruticosa no teste das contorções abdominais induzidas por ácido acético $(0,6 \%)$ em camundongos.

\begin{tabular}{llll}
\hline Grupo & $\begin{array}{l}\text { Dose } \\
(\mathrm{mg} / \mathrm{kg})\end{array}$ & $\begin{array}{l}\text { No. de Contorções } \\
\text { (M. E.P.M) }\end{array}$ & $\%^{{ }^{\mathrm{a}}}$ \\
\hline Controle & - (v.o.) & $20,56 \pm 3,21$ & - \\
& 100 (v.o.) & $18,55 \pm 3,82$ & 9,78 \\
Extrato & 200 (v.o.) & $8,44 \pm 2,02^{*}$ & 58,9 \\
& 400 (v.o.) & $11,44 \pm 2,13^{*}$ & 44,3 \\
Indometacina & 500 (v.o.) & $8,22 \pm 1,36^{* *}$ & 60,0 \\
\hline
\end{tabular}

${ }^{*} \mathrm{p}<0,05 ;{ }^{*} \mathrm{p}<0,01,{ }^{*} * \mathrm{p}<0,001$ (vs controle água; ANOVA, pós-teste de Bonferroni). a. porcentagem de redução do número total de contorções abdominais vs controle. $(\mathrm{n}=9)$.

Tabela 2. Efeito do extrato aquoso da Hyptis fruticosa no teste da formalina (1\%) em camundongos.

\begin{tabular}{llll}
\hline Grupo & Dose (mg/kg) & 1a. Fase & 2a. Fase \\
& & M E.P.M & M E.P.M \\
\hline Controle & - (v.o.) & $66,29 \pm 4,70$ & $50,00 \pm 6,37$ \\
Extrato & 100 (v.o.) & $51,29 \pm 2,50^{*}$ & $65,86 \pm 11,76$ \\
& 200 (v.o.) & $38,57 \pm 4,70^{* * *}$ & $31,86 \pm 12,40$ \\
& 400 (v.o.) & $28,86 \pm 3,82^{* * *}$ & $64,71 \pm 14,80$ \\
Morfina & 7,5 (i.p.) & $8,89 \pm 2,21^{* * *}$ & $0,11 \pm 0,11^{* * *}$ \\
\hline
\end{tabular}

$* \mathrm{p}<0,05 ; * * \mathrm{p}<0,01, * * * \mathrm{p}<0,001$ (vs controle água; ANOVA, pós-teste de Bonferroni). $(\mathrm{n}=7)$.

Tabela 3. Efeito do extrato aquoso da Hyptis fruticosa no teste de placa quente em camundongos.

\begin{tabular}{llll}
\hline Grupo & Dose (mg/kg) & Tempo 0 & Tempo +15 \\
& & M E.P.M. & M E.P.M. \\
\hline Controle & - (v.o.) & $8,51 \pm 0,38$ & $7,97 \pm 0,48$ \\
Extrato & 100 (v.o.) & $10,54 \pm 0,49^{*}$ & $9,00 \pm 0,40$ \\
& 200 (v.o.) & $12,94 \pm 0,57^{* * *}$ & $13,13 \pm 0,38^{* * *}$ \\
Extrato + naloxona & 400 (v.o.) & $7,79 \pm 0,32$ & $7,61 \pm 0,62$ \\
Morfina & 200 (v.o.) +5 (i.p.) & $7,59 \pm 0,77$ & $8,03 \pm 0,77$ \\
Naloxona & 7,5 (i.p.) & $30,00 \pm 0,00^{* * *}$ & $30,00 \pm 0,00^{* * *}$ \\
Morfina + naloxona & 5 (i.p.) & $9,12 \pm 0,96$ & $8,89 \pm 0,97$ \\
\hline
\end{tabular}

$*_{\mathrm{p}}<0,05 ; * *_{\mathrm{p}}<0,01, * * * \mathrm{p}<0,001$ (vs controle água; ANOVA, pós-teste de Bonferroni). $(\mathrm{n}=7)$.

acordo com o esperado, reduziu de maneira significativa a resposta dos animais ao agente nociceptivo em ambas as fases do teste da formalina, enquanto o extrato aquoso $(100,200$ e $400 \mathrm{mg} / \mathrm{kg}$, v.o.) apresentou eficácia apenas na primeira fase deste teste. Estes resultados encontramse na Tabela 2.

Morfina (7,5 mg/kg, i.p.), no teste da placa quente (Tabela 3), aumentou de forma significativa o tempo de latência ao calor. Neste teste, o extrato aquoso da $H$. fruticosa (100 e $200 \mathrm{mg} / \mathrm{kg}$; v.o.) também apresentou efeito antinociceptivo, tendo este efeito sido revertido pelo antagonista opióide naloxona ( $5 \mathrm{mg} / \mathrm{kg}$; i.p.).

No ensaio de toxicidade aguda utilizado, não foi possível calcular a $\mathrm{DL}_{50}$ posto que não foi observada a morte de nenhum animal até 48 horas após a administração oral do extrato aquoso da Hyptis fruticosa (1,3 e 5 $\mathrm{g} / \mathrm{kg}$ ).

\section{DISCUSSÃO}

No presente trabalho, o efeito analgésico, sugerido pelo uso popular para a infusão das folhas da Hyptis fruticosa, foi ratificado nos testes da placa quente, da formalina e das contorções abdominais induzidas por ácido acético.

O teste das contorções abdominais é considerado um modelo de estudo de dor visceral (Gebhart; Ness, 1991). Considerando este fato, a eficácia do extrato aquoso 
da Hyptis fruticosa neste teste sugere que o mesmo seja eficaz no combate a este tipo de dor.

Por outro lado, o teste da formalina (1\%) representa um modelo de estudo de dor moderada e tônica (Hunskaar et al., 1985). Este teste apresenta duas fases distintas, as quais refletem dois tipos diferentes de dor. A primeira fase tem sido atribuída ao efeito direto e imediato do agente flogístico sobre fibras aferentes nociceptivas (Abbadie et al., 1997), enquanto a segunda fase representa um tipo de dor inflamatória (Hunskaar et al., 1985; Hunskaar; Hole, 1987). Fármacos antiinflamatórios, esteroidais e não-esteroidais, reduzem a resposta dos animais apenas na segunda fase do teste da formalina, sendo exceções o ácido acetilsalicílico e o paracetamol, os quais são eficazes em ambas as fases (Hunskaar; Hole, 1987). No presente estudo, o extrato aquoso da Hyptis fruticosa apresentou eficácia apenas na primeira fase do teste da formalina, divergindo, portanto, do padrão de eficácia dos fármacos antiinflamatórios convencionais.

Este padrão de efeito difere também do observado para o extrato aquoso da Hyptis pectinata, o qual apresentou eficácia apenas na segunda fase do teste da formalina (Bispo et al., 2001). Diferentes metabólitos secundários presentes nas duas espécies de Hyptis devem estar colaborando para a variação de padrão de efeito observada.

O teste da placa quente é considerado um teste sensível a fármacos que atuam em nível supraespinal de modulação da resposta dolorosa (Yaksh; Rudy, 1977). Neste modelo de estudo, a eficácia do extrato aquoso da Hyptis fruticosa sugere uma ação modulatória. E, considerando que o efeito analgésico do extrato aquoso, neste teste, foi revertido pelo antagonista opióide naloxona, torna-se razoável sugerir que a analgesia detectada envolva a ativação do sistema opióide. Contudo, este efeito parece não envolver diretamente a ativação dos receptores opióides uma vez que agonistas opióides, diferentemente do extrato aquoso da Hyptis fruticosa, são eficazes em reduzir a resposta dos animais nas duas fases do teste da formalina (Hunskaar; Hole, 1987). O mecanismo exato da ativação do sistema opióide pelo extrato aquoso da $H$. fruticosa permanece por ser estabelecido.

De acordo com Lorke (1983), substâncias cuja $\mathrm{DL}_{50}$ encontra-se acima de $5 \mathrm{~g} / \mathrm{kg}$ são consideradas de baixa toxicidade e os resultados obtidos com doses acima deste valor são considerados imprecisos e de pouco valor prático. $\mathrm{O}$ extrato aquoso da Hyptis fruticosa, no modelo de estudo utilizado, pôde ser considerado um extrato de baixa toxicidade, posto que não foi detectada a morte de nenhum animal com doses de até $5 \mathrm{~g} / \mathrm{kg}$.

Com base nos resultados obtidos concluise que o extrato aquoso da Hyptis fruticosa apresenta efeito antinociceptivo em camundongos e não apresenta toxicidade aguda pelo método utilizado. Estes resultados justificam o uso medicinal desta planta no combate à dor.

\section{AGRADECIMENTOS}

Os autores agradecem ao CNPq e a FAP-SE pelos auxílios financeiros.

\section{REFERÊNCIAS}

Abbadie C, Taylor BK, Peterson MA, Basbaum AI 1997. Differential contribution of the two phases of the formalin test to the pattern of c-fos expression in the rat spinal cord: Studies with remifentanil and lidocaine. Pain 69: 101-110.

Ankier SI 1974. New hot plate tests to quantify antinociceptive and narcotic-antagonist activities. Eur J Pharmacol 27: 1-4.

Barbosa PPP, Ramos CP 1992. Studies on the antiulcerogenic activity of the essential oil of Hyptis mutabilis Briq in rats. Phytother Res 6: 114-115.

Bispo MD, Mourao RH, Franzotti EM, Bomfim KB, ArrigoniBlank MF, Moreno MP, Marchioro M, Antoniolli AR 2001. Antinociceptive and antiedematogenic effects of the aqueous extract of Hyptis pectinata leaves in experimental animals. J Ethnopharmacol 76: 81-86.

Bueno AX, Moreira ATS, Silva FT, Estevam CS, Marchioro M 2006. Effects of the aqueous extract from Hyptis pectinata leaves on rodent central nervous system. Rev Bras Farmacogn 16: 317-323.

Costa JGM, Rodrigues FFG, Angélico EC, Silva MR, Mota ML, Santos NKA, Cardoso ALH, Lemos TLG 2005. Estudo químico-biológico dos óleos essenciais de Hyptis martiusii, Lippia sidoides e Syzigium aromaticum frente às larvas do Aedes aegypti. Rev Bras Farmacogn 15: 304-309.

De Almeida CFCBR, Albuquerque UP 2002. Check-list of the Family Lamiaceae in Pernambuco, Brazil. Braz Arch Biol Technol 45: 343-353.

De Araujo MCM, Cavalcanti MSB, Mello JF, D'Albuquerque IL, Lima O, Delle Monache F, Maciel GM, Lacerda AL 1974. Substâncias antimicrobianas de plantas superiores. Comunicação XLV. Primeiras observações sobre dois novos quinonóides isolados de Hyptis fruticosa Salmz. ex Benth. (Labiatae). Rev Inst Antibiot 14: 101.

De Oliveira CMA, Silva MDR, Kato L, da Silva CC, Ferreira HD, Souza LKH 2004. Chemical composition and antifungal activity of the essential oil of Hyptis ovalifolia Benth. (Lamiaceae). J Braz Chem Soc 15: 756-759.

Delle Monache FD, Marletti F, Marini-Bettolo G, Mello JF, D'albuquerque IL 1977. Diterpenoids of Hyptis fructicosa-(Labiatae). 2. Hyptol. Gazz Chim Ital 107: 319-321.

Dubuisson D, Dennis SG 1977. Formalin test - quantitative study of analgesic effects of morphine, meperidine, and brain-stem stimulation in rats and cats. Pain 4: 161-174.

Falcão HS, Lima IO, Santos VL, Dantas HF, Diniz MFFM, Barbosa-Filho JM, Batista LM 2005. Review of the plants with anti-inflammatory activity studied in Brazil. Rev Bras Farmacogn 15: 381-391.

Gebhart GF, Ness TJ 1991. Central mechanisms of visceral pain. Can J Physiol Pharmacol 69: 627-634. 
Harley RM 1988. Revision of generic limits in Hyptis Jacq (Labiatae) and its allies. Bot J Linn Soc 98: 87-95.

Hunskaar S, Fasmer OB, Hole K 1985. Formalin test in mice, a useful technique for evaluating mild analgesics. $J$ Neurosci Meth 14: 69-76.

Hunskaar S, Hole K 1987. The formalin test in mice - dissociation between inflammatory and noninflammatory pain. Pain 30: 103-114.

Koster R, Anderson M, De Beer EJ 1959. Acetic acid for analgesic screening. Fed Proc 18: 412-416.

Lorke D 1983. A new approach to practical acute toxicity testing. Arch Toxicol 54: 275-287.

Marletti F, Delle Monache F, Marini-Bettolo GB, de Araujo MCM, Cavalcanti MSB, D'albuquerque IL, de Lima OG 1976. Diterpenoid quinones of Hyptis fructicosa (Labiatae). Gazz Chim Ital 106: 119-126.

Morais SM, Dantas JDP, Silva ARA, Magalhães EF 2005. Plantas medicinais usadas pelos índios Tapebas do Ceará. Rev Bras Farmacogn 15: 169-177.

Septimio LR 1994. A fitoterapia baseada em ervas medicinais do C.errado. Brasília: SIPE, Ministério da Cultura.

Souza LKH, Oliveira CMA, Ferri PH, Oliveira JG, Souza AH, Fernandes ODL, Silva MDR 2003. Antimicrobial activity of Hyptis ovalifolia towards dermatophytes. Mem I Oswaldo Cruz 98: 963-965.

Yaksh TL, Rudy TA 1977. Studies on direct spinal action of narcotics in production of analgesia in rat. J Pharmacol Exp Ther 202: 411-428.

Zimmermann M 1983. Ethical guidelines for investigations of experimental pain in conscious animals. Pain 16: 109110. 